\title{
Primary Care Physicians' Knowledge and Practice Patterns in the Treatment of Chronic Kidney Disease: An Upstate New York Practice-based Research Network (UNYNET) Study
}

\author{
Chester H. Fox, MD, Amanda Brooks, MD, Luis E. Zayas, PhD, \\ William McClellan, MD, and Brian Murray
}

Background: There is a steady increase in the prevalence of chronic kidney disease (CKD) in the United States. Primary care physicians (PCPs) can engage in strategies that are proven to be effective in reducing the progression rate of kidney disease. The National Kidney Foundation has released evidencebased guidelines called the Kidney Disease Outcome Quality Improvement Initiative (K/DOQI) that detail these strategies. No information exists regarding adoption of these guidelines in primary care.

Methods: A qualitative study in a practice-based research network (PBRN) was undertaken to explore common PCP practices and knowledge regarding CKD. A typical case sampling strategy was followed. Semi-structured interviews and exit surveys were conducted with 10 PCPs from randomly selected PBRN practices. Three reviewers conducted content analysis using the immersion-crystallization approach.

Results: Five general themes emerged as key findings: (1) lack of awareness of K/DOQI guidelines; (2) Desire for more CKD practice guidance; (3) persistence of traditional, less accurate, diagnostic procedure; (4) variability in the treatment of complications; and (5) uncertainty of timing for referral to a nephrologist.

Conclusion: Facing a growing CKD incidence, PCPs can have an impact on preventing its progression and associated complications with increased familiarity of new guidelines. (J Am Board Fam Med 2006; 19:54-61.)

\section{Background}

Chronic kidney disease (CKD) and end stage renal disease (ESRD) are steadily increasing in both incidence and prevalence in the United States. ${ }^{1,2}$ The number of Americans that have substantial kidney impairment has risen to more than 8 million. An additional 10 million people are estimated to have proteinuria, a potential harbinger of future kidney disease.

Submitted 1 April 2005; revised 1 July 2005; accepted 7 July 2005.

From the Department of Family Medicine (CHF, AB, LEZ), and Department of Medicine and Nephrology (BM), University at Buffalo, Buffalo, NY; and Department of Medicine and Nephrology (WM), Emory University, Atlanta, GA

Funding: This study was funded by a PBRN research stimulation grant from the Joint Grant Awards Program of the American Academy of Family Physicians.

This article is based on a presentation made at the 2005 Convocation of Practices, hosted by the American Academy of Family Physicians National Research Network and the Federation of Practice-based Research Networks, Colorado Springs, CO, March 2005.

Corresponding author: Chester H. Fox, MD, University at Buffalo, Department of Family Medicine, 462 Grider Street, Buffalo, NY 14215 (E-mail: cfox@buffalo.edu).
Epidemiologic studies estimate that there are currently over 20 million people in the United States in various stages of CKD. ${ }^{3-5}$ The incidence of people developing ESRD has increased annually by $6 \%$ to $8 \%$ and is projected to reach over 600,000 people by $2010 .{ }^{6}$ For every patient today who needs renal replacement therapy and has ESRD, there are 20 to 30 patients with some degree of renal impairment who are in danger of progressing to this point. $^{7}$

With the aging of the population and the increasing incidence of obesity, hypertension and diabetes, the number of individuals at risk for CKD will continue to rise. ${ }^{8}$ In 2003, the American Heart Association released a statement that $\mathrm{CKD}$ is a major risk factor for coronary disease. ${ }^{9,10}$ Furthermore, 2 recent studies demonstrate that the risk of death and cardiovascular events in people with coronary artery disease are directly related to the degree of renal dysfunction. ${ }^{11,12}$

Control of hypertension and better glycemic control of diabetes delays progression of CKD to ESRD and death. ${ }^{13,14}$ The use of angiotensin-con- 
verting enzyme inhibitors (ACEI) and Angiotensin receptor blockers (ARB) are particularly effective in protecting the kidneys. ${ }^{15,16}$ Treatment of the anemia that commonly occurs with CKD with erythropoietin-stimulating proteins has also been demonstrated to improve quality of life and delay the progression of the disease. ${ }^{17,18} \mathrm{~A}$ database of unpublished insurance claims data estimates that over $60 \%$ of all patients with CKD are treated exclusively in the primary care office. Using evidencebased guidelines, primary care physicians (PCPs) will be able to detect, monitor, and treat early kidney disease, thereby reducing mortality and improving quality of life for their patients.

The National Kidney Foundation (NKF) has recently released evidence-based guidelines to increase awareness of these secondary prevention strategies in the care of CKD. ${ }^{19}$ In these guidelines, published by the Kidney Disease Outcome Quality Initiative (K/DOQI) Advisory Board, estimated glomerular filtration rate (GFR) is the recommended CKD screening/diagnostic test, as opposed to the more commonly used serum creatinine. ${ }^{13}$ These are the only known evidence-based guidelines for pre-dialysis CKD care (http://www. guidelines.gov); (the Veterans Affairs guideline reads more like a basic "how-to" teaching manual), and they are rigorous in describing the level of evidence and validity of their recommendations.

The estimated GFR has been proven to be a more accurate measure of kidney function than the standard serum creatinine and can be used to both diagnose and stage the severity of $\mathrm{CKD} .^{20}$ The formula involves the age, sex, race, and serum creatinine of the patient. The PDA tool MedCalc contains this formula, and a tool can also be downloaded to a computer or PDA from the National Kidney Disease Education Project (http://www. nkdep.nih.gov/). This website is a very valuable link for the PCP to learn how to diagnose and treat $\mathrm{CKD}$ in an evidence-based manner.

A literature search using the keywords "chronic kidney disease" and "primary care" revealed no articles that describe how well PCPs are doing in incorporating this guideline into practice. Because $\mathrm{CKD}$ is a growing public health issue whose natural history can be changed with early intervention, we decided to conduct a qualitative exploration of common knowledge and practices of PCPs in the diagnosis and treatment of CKD.

\section{Methods}

This study involved individual in-depth interviews with a target sample of 10 PCPs recruited from 10 different practices affiliated with the Upstate New York Practice-based Research Network (UNYNET), which encompasses 50 practices. A "typical case" sampling strategy that "focuses directly on the ordinary and usual," was followed. ${ }^{21}$ A list of all 50 practices was drawn in random order from which to recruit one physician per practice until achieving the desired sample size of 10 , for a $20 \%$ practice site representation. Due to technical difficulties, one of the completed interviews could not be transcribed and was discarded, and another participant was recruited. This sample of UNYNET practices was comprehensive regarding type of practice, urban or rural setting, academic affiliation, and patient populations. The Institutional Review Board of the University at Buffalo approved this study.

A semi-structured in-depth interview guide with 12 open-ended questions and a short exit questionnaire were used to collect data. The questions utilized are presented in the Appendix. A qualitative design was used for exploratory aims to elicit detailed opinions and experiences from PCPs regarding CKD patient care without limiting the scope or content of their responses. The interview's openended format allowed the respondents to elaborate on their answers and offer case examples after or without probing. ${ }^{22} \mathrm{~A}$ research assistant trained in qualitative data collection interviewed all the physicians in their offices for approximately 1 hour. The questions pertained to the physician's definition, diagnostic pattern, monitoring, severity assessment, treatment modalities, knowledge of national guidelines, and referral criteria for CKD. All the interviews were audio recorded and transcribed for analysis. The exit questionnaire was anonymous. It was self administered and filled out by each of the clinicians immediately after the interview.

A family doctor and a nephrologist developed both the interview guide and questionnaire, and a medical anthropologist reviewed the interview questions to insure consistency and appropriateness. A team of three researchers-the family doctor, the research assistant, and the medical anthropologist-analyzed the data following a contentdriven immersion-crystallization approach. ${ }^{23}$ This 
analytical approach involved a systematic iterative process of interpretive review, deliberation, and categorization. First, each analyst independently reviewed each transcript and identified meaningful units of information pertaining to the research query. The analysts then compared their initial findings, deliberated about their significance, and identified patterns leading to emerging themes. Themes were supported by multiple references and derived by consensus. A data set search for conflicting evidence was performed as a means to support the trustworthiness of the findings. ${ }^{24,25}$ Questionnaire data were entered into a database for descriptive statistical analysis.

\section{Results}

The respondents varied in location, specialty, and academic affiliation. The $10 \mathrm{PCPs}$ interviewed came from a wide radius from Buffalo, NY (Figure 1). There were 7 family physicians and 3 internists, 2 academic and 8 community physicians, and 2 urban, 4 suburban, and 4 rural physicians. There was a wide geographic spread, 29 miles and 3 separate counties; a mix of urban, suburban, and rural practices; a mix of community and academic practices; and both internal medicine and family medicine were represented. "This constitutes $20 \%$ of our network practices and is fairly representative of the remaining 40 practices in the network regarding these characteristics.

Qualitative study findings are presented below in narrative form under the following general themes: (1) lack of awareness of evidence-based guidelines for CKD; (2) desire for more CKD practice guidance; (3) persistence of traditional, less accurate, diagnostic procedure; (4) variability in the treatment of complications; and (5) uncertainty of timing for referral to a nephrologist.

\section{Lack of Awareness of Evidence-based Guidelines}

None of the physicians interviewed reported that they regularly used or were aware of the NKF's evidence-based K/DOQI guidelines. One physician specifically stated: "I'm not aware of any

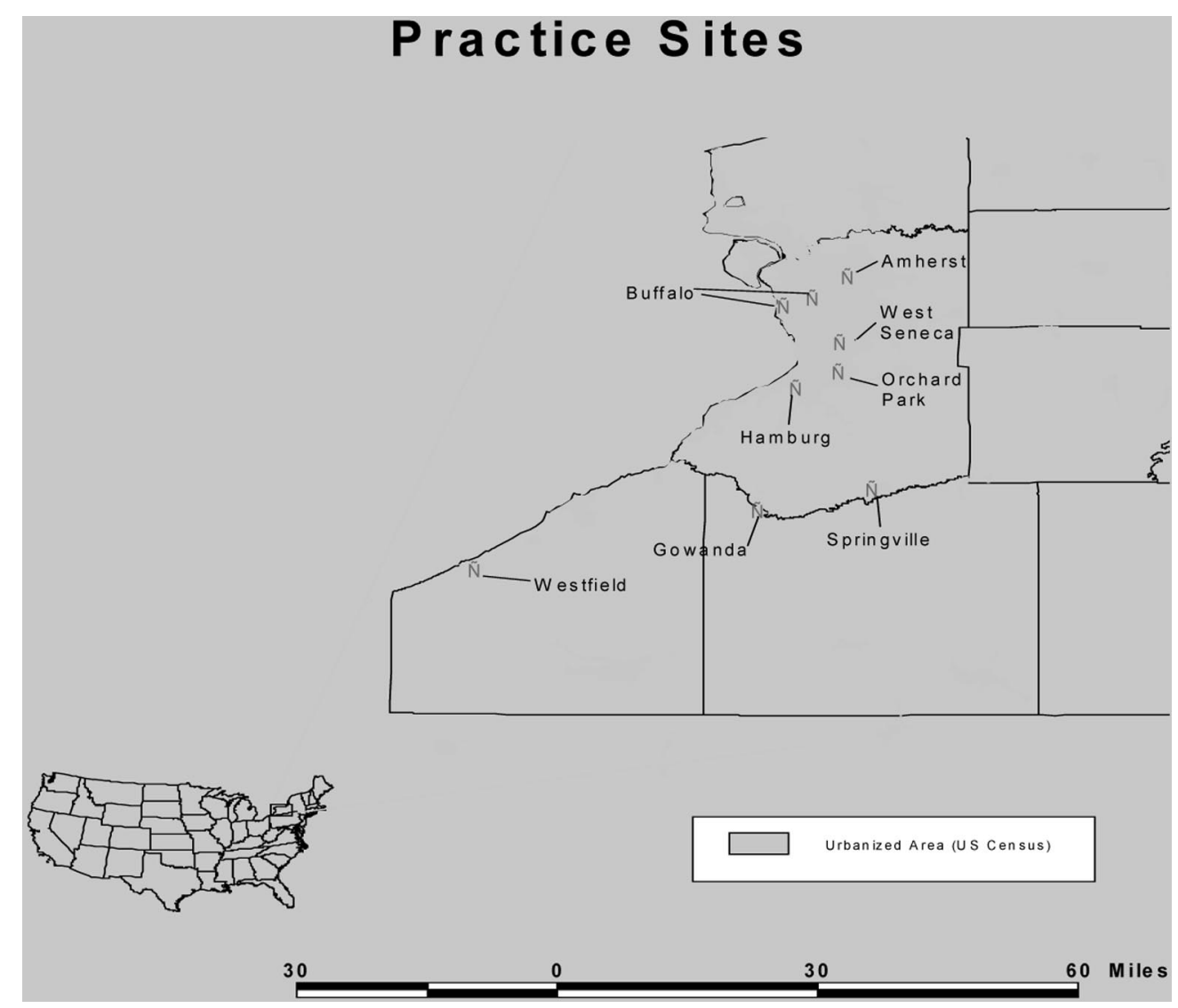

Figure 1. Practice sites in the 8 counties where the interviews were conducted. 
guidelines" for CKD. The key points of the K/DOQI guidelines and the data on whether or not the 10 physicians understood and/or practiced these guidelines are summarized in Table 1. This table compares the qualitative and quantitative interview results.

\section{Desire for More CKD Practice Guidance}

One physician admitted that the lack of well-known guidelines in CKD care has led kidney disease to be "an area that I believe that many of us practice, I don't know, by hook or by crook." More than half expressed a favorable opinion of the use of guided care. In fact, the large majority of the cohort voiced a desire for better guidance in treating kidney disease. "I think that if you guys come up with some type of guidelines that would help us better to manage $[\mathrm{CKD}]$ in our office that would be great."

In daily practice, all 10 of the physicians admitted using the guidelines for diabetes and hypertension and felt that these guidelines were adequate in diagnosing and treating kidney disease. As one physician noted: "I think a lot of family physicians don't think about chronic kidney disease the way we think about some of the other guidelines for hypertension and diabetes, so that would be good to be brought to the forefront."

\section{Persistence of Traditional, Less Accurate, Diagnostic Procedure}

There was little variation in the physicians' preferences for kidney disease diagnostic tests: serum creatinine was the test of choice. All but one of the physicians interviewed use blood urea nitrogen (BUN), serum creatinine (Cr), and urinalysis as standard screening protocol. Only one physician reported regular use of calculated GFR. This respondent stated that he presently uses this diagnostic test because his laboratory reports the result routinely as a standard feature of a metabolic profile.

Although most physicians are using serum creatinine, a few did emphasize the importance of using a more accurate test. Two physicians mentioned the use of creatinine clearance instead of serum creatinine to more accurately measure kidney function. Both said that they did not use creatinine clearance in regular practice because of the inconvenience of using a 24-hour urine test. "I usually follow more with creatinine than creatinine clearance which is probably not the best thing to do." Although creatinine clearance is a more accurate test than serum creatinine for diagnosing $\mathrm{CKD}$, it is not very practical in clinical practice. The estimated GFR gives improved accuracy without the inconvenience.

\section{Variability in the Treatment of Complications}

Of the many complications of CKD, physicians voiced the greatest concern for cardiovascular problems. All the physicians mentioned peripheral vascular disease, hypertension, congestive heart failure, and coronary artery disease as important issues in CKD care.

All the participants emphasized the importance of aggressively treating the comorbidities of kidney

Table 1. Physician Practice Patterns Related to the Key Points of the K/DOQI Guidelines ${ }^{29,30}$

\begin{tabular}{lcc}
\hline Guideline & Qualitative Interview $(\mathrm{n}=10)$ & Quantitative Exit Survey $(\mathrm{n}=10)$ \\
\hline Use of GFR to diagnose CKD & 1 & N/A \\
Check for microalbumin & N/A & 9 \\
Control BP to $<130 / 80$ & 10 & 10 \\
Use ACE/ARB early & 8 & N/A \\
Treat cholesterol & & 8 \\
HDL $>40$ & & \\
LDL $<100$ & 2 & N/A \\
Use erythropoietin for anemia & 1 & 1 \\
Keep hemoglobin $>11$ & 2 & 8 \\
Check calcium, phosphorous, and PTH for bone disease & 0 & N/A \\
Refer to nephrologist for GFR $<30$ & &
\end{tabular}

K/DOQI, Kidney Disease Outcomes Quality Initiative; GFR, glomerular filtration rate; CKD, chronic kidney disease; BP, blood pressure; ACE, angiotensin-converting enzyme; ARB, angiotensin receptor blocker; HDL, high-density lipoprotein; LDL, lowdensity lipoprotein; PTH, parathyroid hormone. 
disease especially hypertension, diabetes, and hyperlipidemia.

In the treatment of hypertension, almost the entire cohort advocated using ACEIs in patients with renal failure, especially if they had diabetes. However, one physician reported sometimes avoiding use of ACEIs: "I tend to be more cautious with diuretic and... don't use ACE inhibitors as readily."

On the topic of anemia, less than half knew the importance of recognizing anemia, and only two regularly prescribed erythropoietin or darbepoietin. Many of the physicians left it up to a nephrologist to begin treatment with erythropoietin replacement. This usually occurred later in the course of the disease. None of the physicians reported referring a patient to a nephrologist specifically for the treatment of anemia even when they did not treat it themselves.

Most of the physicians did not recognize secondary hyperparathyroidism and bone disease as associated with CKD. Only two of ten reported routine testing of phosphate and parathyroid hormone in their patients with CKD.

Most of the physicians also reported avoiding nonsteroidal anti-inflammatory drugs (NSAIDs) in pain management due to the renal damage they can cause. However, a few admitted to giving NSAIDs to CKD patients: “Let's say some patients you can't give nonsteroidal to and others it doesn't seem to bother them that much."

\section{Uncertainty of Timing for Referral to a Nephrologist}

There was uncertainty about when to refer to a nephrologist. One respondent, for example, refers to a nephrologist at "a creatinine clearance below 20 ," another refers at "a creatinine of 1.2," and a third one admitted to referring after reaching the point "when I'm not comfortable anymore." There was no consistent basis for or reasoning about the timing of referral.

The majority identified communication with the specialist as problematic. As one physician complained: "I get frustrated with consultants who don't recognize that [lack of communication] because patients are coming to me for information and if the consultant doesn't coordinate with me then the patient either gets poor care, poor understanding of their disease or frustrated."
Some rural physicians expressed difficulty in patient access to care. One of them noted, "I have difficulty in getting my patient to the specialist. They are unavailable and far away. I try to manage as much as I can myself."

Many voiced confusion over the PCP's role once a CKD patient is under the care of a specialist. In most cases, once referred, the specialist tended to take over the care of the patient entirely: "some of them are just better about communicating than others, so we end up sometimes trying to manage these patients without all the information we need to manage them. That's a big area of disconnect for us. . " Many expressed not knowing whether they or the specialist was responsible for various types of screening and care. For example, it was often not clear who was responsible for the hypertension, diabetes, and lipid management. Also there needed to be clarification as to who would order which laboratory tests. If the PCP and the nephrologist did not use the same information system, then laboratory results were often not shared in a timely fashion.

Only a few of the physicians said they worked together well with a nephrologist: "patients continue to follow here regularly and I feel free to alter medications and that kind of thing, especially in relation to diabetes, hypertension, coronary disease,... If there's a kidney specific issue then that often gets deferred to the nephrologist." PCPs expressed greater satisfaction with the relationship when they had a nephrologist in their practice group or a specific nephrologist to whom they always referred patients.

\section{Discussion}

There is an urgent need to increase the PCP's awareness of the evidence-based CKD care in the $\mathrm{K} / \mathrm{DOQI}$ guidelines. There is also an educational need to present the key elements of these guidelines in an unambiguous format so that PCPs can rapidly incorporate them into their daily practice.

Although all the physicians studied were aware of and using guidelines for hypertension and diabetes, all were unaware of the existence of CKD guidelines. There are a number of reasons for this. First, guidelines for hypertension and diabetes are older and better publicized. The K/DOQI guidelines are relatively new, and some are still being developed. In addition, PCPs generally see more 
hypertensive and diabetic patients in their practice than CKD patients. A third barrier is that guidelines are often cumbersome to read. The K/DOQI guidelines are over 200 pages, and competing demands make it difficult for the PCP to assess what is practical in the routine care of patients. ${ }^{26}$ Both JNC VII guidelines for hypertension and the Adult Treatment Panel III (ATP III) guidelines for cholesterol have very concise 1-page tables that describe the suggested procedures for various stages of the disease. ${ }^{27,28}$

Based on these guidelines, there are presently a number of procedures that will go a long way toward delaying progression to ESRD and improving quality of life for our patients. The key points that will help the PCP improve their diagnosis and treatment of CKD are outlined in Table 2.

Most of the physicians were not aware of using GFR to diagnose CKD. One simple solution could be as follows: once the physician has been made aware of this diagnostic tool, they should request that the laboratory automatically report the GFR whenever a serum creatinine is ordered. This is currently done by all hospitals in western New York. In the meantime, physicians should remember that GFR is influenced by race, sex, and age.

Most patients with CKD have multiple comorbid conditions. This makes it difficult for the PCP to know when to refer a patient and how to set up clear lines of communication with the specialist. Many of these patients are seeing multiple specialists. Maximizing the PCP's ability to diagnose and treat the complications of early CKD (stages 1 to 3 ) will allow for easier access to appropriate specialty care. The Renal Physician's Association developed a template letter between PCP and specialist that helps to standardize the communication and the roles of the various care providers. The letter is available at http://www.niddk.nih.gov/forms/nkdep/template.asp. Greater dissemination and more research into the testing of these types of devices are needed for improved primary care/specialty collaboration for improved patient care.

The limitations of this study are the small sample size, and the relatively narrow geographic area. Either a broader national survey or a repeat of this study in other areas of the country would be needed to more fully validate our conclusions. Despite these limitations, our hope is that clinicians will take prompt action to improve CKD patient care.

\section{Next steps}

As a result of this study, a couple of the next steps were developed. After reviewing the findings, it was felt that a broader survey needed to be done to test the generalizability of this study. A 36 question web based survey was developed by the investigative team and it will be pilot tested in two PBRNs in New York and Wisconsin. If the survey proves useful in this pilot phase, a national survey may be undertaken.

The results of this study were presented at a number of regional NKF Chronic Kidney Disease Symposia. It was felt that the two key findings were the educational need of the PCP as to how to recognize and treat $\mathrm{CKD}$ and the poor communication between the PCP and specialist. This was discussed at our western New York kidney disease collaborative, of which the regional NKF is a member. It was felt that there needed to be face to face discussions between PCP and specialist as to how to improve these 2 areas. The regional NKF is sponsoring a series of roundtable discussions with 5 nephrologists and 10 PCPs from our 8-county re-

\section{Table 2. Key Points to Improve Diagnosis and Treatment of Chronic Kidney Disease 26,27,28 $^{2}$}

- Estimated GFR is more accurate than serum creatinine in the diagnosis of CKD.

- A GFR of $<60$ puts a patient at risk for the complications of CKD, which are coronary artery disease, anemia, and bone loss due to secondary hyperparathyroidism.

- When the GFR falls below 60, stop metformin and all nonsteroidals and COX-2 inhibitors.

- Angiotensin-converting enzyme inhibitors or angiotensin receptor blockers slow the progression of CKD.

- CKD is a coronary artery disease equivalent; therefore, lipid goals should be LDL $<100$ and HDL >40.

- If the hemoglobin is $<11$ due to CKD, then erythropoietin replacement will reduce mortality, delay progression to dialysis, and improve quality of life.

- Checking calcium, phosphorous, and PTH will help secondary hyperparathyroidism. Treatment to keep the calcium phosphate product below 55 and keeping the PTH as close to normal as possible will help prevent bone loss and delay the progression of the disease.

GFR, glomerular filtration rate; CKD, chronic kidney disease; LDL, low-density lipoprotein; HDL, high-density lipoprotein; PTH, parathyroid hormone. 
gion. The goal of this roundtable is to create a 1-page educational tool that summarizes key evidence-based points of the K/DOQI guidelines and to give advice on when referral to a specialist should be considered. The second goal is to clearly delineate roles and responsibilities of PCP and specialist once a patient is referred. Communication about who writes which drugs and who orders which laboratory tests and how information is shared is the focus of these discussions.

\section{Appendix A-Interview Guide and Exit Questionnaire}

PCP's Knowledge and Treatment of Chronic Kidney

\section{Disease}

Principal Research Question: What are the knowledge and practice patterns of PCPs regarding the detection and treatment of kidney disease and its complications? Are opportunities for the delaying of the progression of the disease being missed?

1. How do you define chronic kidney disease (CKD)?

2. How do you describe this illness to your patients?

3. How do you diagnose it?

4. How do you monitor it?

5. How do you judge the severity of the disease?

6. What is the time line and prognosis for patients with $\mathrm{CKD}$ ?

7. What are some comorbidities and complications of CKD?

8. How do you treat the complications of CKD?

9. Please explain whether or not you modify your treatment of other diseases (eg, hypertension, hyperlipidemia, diabetes, etc) in patients with CKD?

10. Please explain whether or not you follow national guidelines for treating CKD?

11. What are your criteria for referring patients with CKD to a nephrologist?

12. Is there anything else you want to say about your understanding or treatment of CKD?

\section{Exit Questionnaire}

1. What is your medical specialty?

2. For how many years have you been practicing this specialty?
3. Approximately what percentage of the patients that you see is of the following ethnic backgrounds:

\% European American

$\%$ African American

$\%$ Latino

$\%$ Other

4. Approximately how many patients do you treat for CKD?

5. What is the ethnic background of the majority of your patients with CKD?

6. Approximately what is the gender distribution of your patients with CKD? _ \% $\mathrm{M}$; _ _ $\% \mathrm{~F}$

7. What would you consider good blood pressure control in a patient with CKD? (Circle answer) $140 / 90 \quad 130 / 85 \quad 125 / 75 \quad$ Other

8. What level of LDL should be targeted in patients with CKD and no clinical evidence of CAD? $160 \quad 130 \quad 100$ Other

9. What level of HgbA1C should be targeted in patients with CKD? $7.0 \quad 8.0 \quad 9.0 \quad$ Other

10. Do you check microalbumins? Yes/No

11. How often do you check for proteinuria in patients with diabetes? 3 months 6 months Yearly Never Other

12. How often do you check for proteinuria in patients with hypertension? 3 months 6 months Yearly Never Other

13. How often do you check a serum creatinine in patients with diabetes and/or hypertension?

3 months 6 months Yearly Never Other

14. At what creatinine level would you stop using ACE inhibitors or ARBs in CKD?

$\begin{array}{lllll}2.0 & 2.5 & 3.0 & \geq 3.5 \quad \text { Never }\end{array}$

15. At what hemoglobin level would you prescribe erythropoietin in patients with $\mathrm{CKD}$ ?

$\leq 9.0 \quad 10.0 \geq 11.0 \quad$ Never

16. Do you ever order a parathyroid hormone level in patients with CKD? Yes/No

We thank Renee Cadzow, MA, for help in editing this manuscript.

\section{References}

1. Hostetter TH, Lising M. National kidney disease education program. J Am Soc Nephrol 2003;14: S114-6.

2. Nickolas TL, Frisch GD, Opotowsky AR, Arons R, Radhakrishnan J. Awareness of kidney disease in the 
US population: findings from the National Health and Nutrition Examination Survey (NHANES) 1999 to 2000. Am J Kidney Dis 2004;44:185-97.

3. St Peter WL, Schoolwerth AC, McGowan T, McClellan WM. Chronic kidney disease: issues and establishing programs and clinics for improved patient outcomes [Review]. Am J Kidney Dis 2003;41:90324.

4. Kausz ATMM, Levey ASM. The care of patients with chronic kidney disease: what must we do and who should do it? [Editorial]. J Gen Intern Med 2002;17:659-63.

5. Hsu CY, Vittinghoff E, Lin F, Shlipak MG. The incidence of end-stage renal disease is increasing faster than the prevalence of chronic renal insufficiency. Ann Intern Med 2004;141:95-101.

6. Levey AS, Coresh J, Balk E, et al. National Kidney Foundation Practice Guidelines for chronic kidney disease: evaluation, classification, and stratification. Ann Intern Med 2003;139:137-47.

7. Parmar MS. Chronic renal disease. BMJ 2002;325: 85-90.

8. Wetterhall SF, Olson DR, DeStefano F, et al. Trends in diabetes and diabetic complications, 1980-1987. Diabetes Care. 1992;15:960-7.

9. Coresh J, Astor B, Sarnak MJ. Evidence for increased cardiovascular disease risk in patients with chronic kidney disease. Curr Opin Nephrol Hypertens 2004; 13:73-81.

10. Sarnak MJ, Levey AS, Schoolwerth AC, et al. Kidney disease as a risk factor for development of cardiovascular disease: a statement from the American Heart Association Councils on Kidney in Cardiovascular Disease, High Blood Pressure Research, Clinical Cardiology, and Epidemiology and Prevention. Hypertension 2003;42:1050-65.

11. Go AS, Chertow GM, Fan D, McCulloch CE, Hsu CY. Chronic kidney disease and the risks of death, cardiovascular events, and hospitalization. N Engl J Med 2004;351:1296-305.

12. Anavekar NS, McMurray JJ, Velazquez EJ, et al. Relation between renal dysfunction and cardiovascular outcomes after myocardial infarction. N Engl J Med 2004;351:1285-95.

13. Johnson DW. Evidence-based guide to slowing the progression of early renal insufficiency. Intern Med J 2004;34:50-7.

14. Lameire N, Stevens P, Raptis S, Thomas S, Schernthaner $\mathrm{G}$. Individualized risk management in diabetics: how to implement best practice guidelines-design and concept of the IRIDIEM studies. Kidney Blood Press Res 2004;27:127-33.

15. Snively CS, Gutierrez C. Chronic kidney disease: prevention and treatment of common complications. Am Fam Physician 2004;70:1921-8.

16. Boos C. Cardiovascular protection with ace inhibitors-more HOPE for EUROPA? Med Sci Monit 2004;10:SR23-8.
17. Gouva C, Nikolopoulos P, Ioannidis JP, Siamopoulos KC. Treating anemia early in renal failure patients slows the decline of renal function: a randomized controlled trial. Kidney Int 2004;66:753-60.

18. Silverberg DS, Wexler D, Iaina A. The role of anemia in the progression of congestive heart failure. Is there a place for erythropoietin and intravenous iron? J Nephrol 2004;17:749-61.

19. Johnson CA, Levey AS, Coresh J, Levin A, Lau J, Eknoyan G. Clinical practice guidelines for chronic kidney disease in adults: part I. definition, disease stages, evaluation, treatment, and risk factors. Am Fam Physician 2004;70:869-76.

20. Levey AS, Bosch JP, Lewis JB, Greene T, Rogers N, Roth D. A more accurate method to estimate glomerular filtration rate from serum creatinine: a new prediction equation. Modification of diet in renal disease study group. Ann Intern Med 1999;130:46170 .

21. Kuzel AJ. Sampling in qualitative research. In: Crabtree BF, Miller WL, editors. Doing qualitative research. Thousand Oaks (CA): Sage Publications, Inc.; 1999. p. 33-45.

22. Miller WL, Crabtree BF. Depth interviewing. In: Crabtree BF, Miller WL, editors. Doing qualitative research. Thousand Oaks (CA): Sage Publications, Inc.; 1999. p. 89-107.

23. Borkan J. Immersion/crystallization. In: Crabtree B, Miller $W$, editors. Doing qualitative research. Thousand Oaks (CA): Sage Publications, Inc.; 1999. p. 20-4.

24. Miller WL, Crabtree BF. Primary care research: a multimethod typology and qualitative road map. In: Miller WL, editor. Doing qualitative research. Newbury Park (CA): Sage Publications, Inc.; 1992. p. $3-28$.

25. Kuzel AJ, Like RC. Standards of trustworthiness for qualitative studies in primary care. In: Dunn EV, editor. Primary care research: traditional and innovative approaches. Vol. 1. Newbury Park (CA): Sage Publications, Inc.; 1991. p. 138-158.

26. Jaen CR, Stange KC, Nutting PA. Competing demands of primary care: a model for the delivery of clinical preventive services. J Fam Pract 1994;38: 166-71.

27. Chobanian AV, Bakris GL, Black HR, et al. Seventh report of the Joint National Committee on Prevention, Detection, Evaluation, and Treatment of High Blood Pressure. Hypertension 2003;42:1206-52.

28. Lauer MS, Fontanarosa PB. Updated guidelines for cholesterol management. JAMA 2001;285:2508-9.

29. K/DOQI clinical practice guidelines on hypertension and antihypertensive agents in chronic kidney disease. Am J Kidney Dis 2004;43:S1-290.

30. Al Aly Z, Gonzalez EA, Martin KJ, Gellens ME. Achieving K/DOQI laboratory target values for bone and mineral metabolism: an uphill battle. Am J Nephrol 2004;24:422-6. 